\title{
A NESTED CORTEX PARCELLATION COMBINING ANALYSIS OF MEG FORWARD PROBLEM AND DIFFUSION MRI TRACTOGRAPHY
}

\author{
Anne-Charlotte Philippe, Maureen Clerc, Théodore Papadopoulo, Rachid Deriche \\ Athena Project-Team, INRIA Sophia Antipolis - Méditerranée, France
}

\begin{abstract}
Understanding the relationship between structure and function is a major challenge in neuroscience. Diffusion MRI (dMRI) in the only non-invasive modality allowing to have access to the neural structure. Magnetoencephalography (MEG) is another non-invasive modality that allows a direct access to the temporal succession of cognitive processes. Functional cortex parcellation being one of the most important ways to understanding structure-function relationship, we propose an innovative method merging MEG and dMRI to parcellate the cortex. The combination of MEG forward problem and connectivity information reveals cortical areas generating a similar magnetic field at sensors while having a similar connectivity. Results show suitable clusters that forecast interesting studies for inter- and intra- subjects comparisons of the cortex parcellations. The automatic nested cortex parcellation we propose could be a first step to analyse sources that are seeds of long or short range connectivity and to differentiate these connectivities in the white matter.
\end{abstract}

Index Terms - MEG, dMRI, cortex parcellation

\section{INTRODUCTION}

It has been demonstrated that cyto- and myeloarchitectonic areas show a clear functional segregation [1]. Thus, it is essential to study the correspondence between structural and functional cortex parcellation. Studying this neuroscientific problem through at the same time a structure-based modality and a function-based modality, we could improve the understanding of the link between structures of the brain and cognitive processes.

Diffusion MRI (dMRI) is the only non-invasive modality allowing to have access to the architecture of neural tissues [2]. This is why an increasing number of papers exploits diffusion MRI tractography to parcellate the cortex for a better understanding of the functional activity in the brain [3][4][5] but connectivity-based parcellation is a computationally intensive problem which has to be intensively reduced [5] or limited to an area well-known functionally [3][4] .

Besides, magneto-encephalography (MEG) is a noninvasive modality giving more direct access to the temporal succession of cognitive processes. The localization of sources on the cortex responsible of MEG measurements, i.e the inverse problem, relies on the solution of the MEG forward problem linking the neural current of cortical sources to the scalp. The inverse problem is under-constrained having a huge number of cortical sources compared to the number of measurements. Cortex parcellation for MEG allows to consider a smaller number of sources for the inverse problem.

Some studies have used dMRI for analyzing the connectivity of sources having an abnormal activity through MEG [6][7]. The objective of the present paper was to combine both MEG forward problem and diffusion MRI tractography to improve cortex parcellation. Thus, we take advantage of the MEG forward problem to reduce the number of sources in order to perform the computationally intensive problem of connectivity-based parcellation. Besides, we facilitate MEG analysis by reducing the number of cortical regions detectable by the MEG inverse problem, while keeping the connectivity information. This paves the way for a functional connectivity analysis in MEG informed by structural connectivity, by introducing spatio-temporal constraints in the inverse problem.

\section{METHODOLOGY}

\subsection{Image acquisition}

MRI acquisition was performed using a 3 Tesla Siemens scanner with a 12-channel head coil. First, a 3D T1 weighted image $\left(\mathrm{TR} / \mathrm{TE}=2.2 \mathrm{~s} / 2.94 \mathrm{~ms}\right.$, flip angle $=10^{\circ}$, matrix size $=128 \times 128$, 208 axial slices, voxel size $=1 \times 1 \times 1 \mathrm{~mm}^{3}$ ) was collected. A set of diffusion-weighted images (DWI) (TR/TE $=12 \mathrm{~s} / 86 \mathrm{~ms}$ flip angle $=90^{\circ}$, one average, 60 contiguous axial slices, voxel size $2 \times 2 \times 2 \mathrm{~mm}^{3}, 64$ gradient directions, $b$-value $=1000 \mathrm{~s} / \mathrm{mm}^{2}$ ) was acquired with five B0 images and no diffusion gradient.

\subsection{Image processing}

\subsubsection{Registration and Surface extraction}

We worked in the diffusion space. We linearly registered the T1 weighted MRI on the space of study with FSL ${ }^{1}$. Several head surfaces were extracted using FreeSurfer ${ }^{2}$ with the registered T1 weighted MRI. We meshed the inner skull, outer

\footnotetext{
${ }^{1}$ www.fmrib.ox.ac.uk/fsl/

${ }^{2}$ surfer.nmr.mgh.harvard.edu
} 


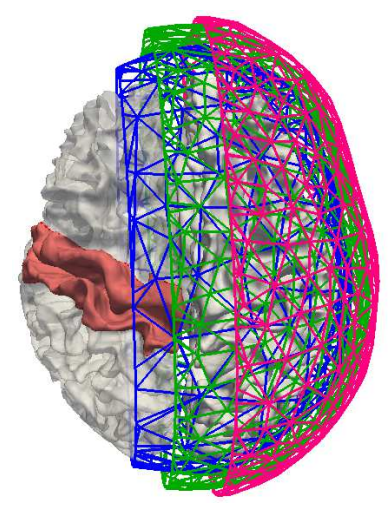

Fig. 2. Surface meshes: inner skull (blue), outer skull (green), skin (pink) surface, WM/GM interface (grey), ROI (red).

skull and skin surfaces with a coarse resolution (around 600 vertices per mesh) and the interface between the white matter (WM) and the grey matter (GM) with a high resolution (around 100000 vertices per mesh, one mesh by hemisphere). All these meshes are presented on Fig. 2. We simulated positions of 303 magnetometers outside of the scalp.

\subsubsection{Region of Interest}

We studied the two gyri of the WM/GM interface on both sides of the left central fissure, represented as the red part of the WM/GM boundary mesh on Fig. 2. This region of interest (ROI) involves a lot of functional connections and structural connectivities. The mesh comprised 11796 vertices. We placed on each vertex an electric dipole with a fixed orientation normal to the mesh, directed toward the outside of the mesh. The same location was used to place a seed for the tractography.

\subsection{MEG forward problem}

The MEG forward problem concerns the calculation of magnetic fields at sensor positions given the neural sources in the brain. It depends on the position, the orientation and the magnetic intensity of distributed sources.

We computed the lead field matrix $G$ with OpenMEEG ${ }^{3}$ using a conductivity value in the brain of 1 , in the skin of 1 , in the air of 0 and in the skull of 0,03 . Thus, $G(i, j)$ relates the magnetic field generated by the $j$-th source observed at the $i$-th sensor. The relationship between the measurements $M$ on MEG sensors and the cortical activity $S$ is modeled by $M(t)=G S(t)+N$ where $N$ represents a Gaussian centered white noise.

Considering a distributed sources model on the ROI, the MEG inverse problem is obviously under-constrained: we have to determine the intensity of 11796 sources with only

\footnotetext{
${ }^{3}$ gforge.inria.fr/projects/openmeeg/
}

303 measurements on sensors. Different methods exist to solve this problem. The Minimum-Norm methods selects the source activity $S$ that minimizes the difference between $M(t)$ and the ideal measurements $G S(t)$, i.e $\min _{S} \| M(t)-$ $G S(t) \|_{2}^{2}$. These methods need a regularization on $S$ to stabilize the solution(Tikhonov regularization, $L 2$ norm).

Our nested cortex parcellation is a new way to constrain $S$ : combining together sources that generate a similar magnetic field at sensors while having a similar connectivity, they can represented as a single source without losing cortical activity information nor structural information.

\subsection{White Matter Probabilistic Tractography and con- nectivity profile of a source}

Two families of tractography algorithms exist: deterministic and probabilistic algorithms. Deterministic WM fiber tractography is most commonly implemented using the principal diffusion direction of the estimated diffusion tensor. The main limitation of deterministic tractography is that it generates fibers following only one direction of diffusion per voxel, so algorithms are very sensitive to the estimated principal direction. Probabilistic algorithms are computationally more expensive than deterministic algorithms but can better deal with partial volume averaging effects and noise uncertainty in underlying fiber directions. Most importantly, the output of the probabilistic algorithms is usually a connectivity index measuring how probable it is that two voxels are connected. Hence, taking each voxel of the WM as a target, we can have a good overview of the degree of connectivity between the seed and all voxels of the WM. In addition, contrary to deterministic methods which privilege long range connectivity, probabilistic tractography can detect fibers of short-range connectivity, typically fibers of the U system [8]. For all these reasons, we decided to use probabilistic tractography in our approach.

We used FSL to compute the connectivity image of each source taking all voxels of the WM as targets. All voxels of the output image have a value (though many of these will be zero) representing the connectivity value between the source and that voxel, see Fig. 3. We reshaped each connectivity image into a vector called the connectivity profile $C P_{i}$ of the source $i[9]$.

\subsection{Cortex Parcellation}

Two sources of information are known: the lead field and the $C P$. The idea behind our cortex parcellation illustrated on the flowchart on Fig. 1 is to mark the boundary of sources the leadfield can not distinguish. Then, lead field-based parcellation is refined delineating sources having a similar connectivity.

Sources which can not be separated by the MEG inverse problem are ones generating close magnetic fields on all sensors. Thus, we computed the MEG lead field of sources as 
IMAGE PROCESSING

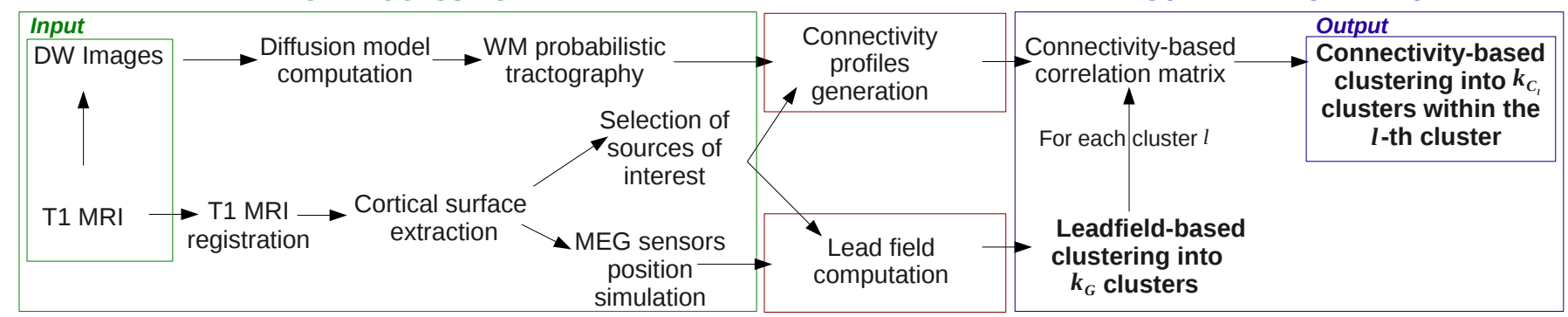

Fig. 1. Computational processing flowchart for the nested cortex parcellation method.

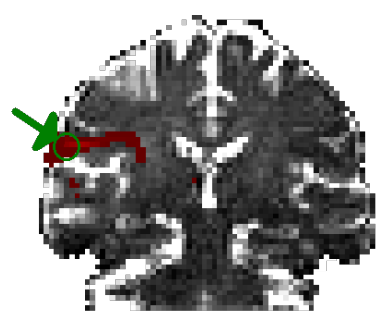

Fig. 3. Connectivity image (in red gradation corresponding to the degree of connectivity) of a cortical source (indicated in green), the background in grey levels is a coronal slice of a DW Image.

in 2.3 within the ROI defined section 2.2.2. Let us recall that the $j$-th column of this lead field concerns the magnetic field generating by the $j$-th source on all sensors. That is why, to group sources generating a close magnetic field on all sensors, we applied a $k$-means algorithm to the columns of the lead field, Algorithm 1: ligne 3. This produces a set of $k_{G}$ clusters, grouping sources that cannot be separated on the basis of MEG.

Then we included connectivity information, given by the connectivity profiles of sources Algorithm 1: line 4 to 9 . For each source within a lead field-based cluster, we computed the correlation values between any two of the connectivity profiles as described in 2.4 and arranged them into a connectivity-based correlation matrix $\operatorname{corr} C P_{l}$, Algorithm 1: line 5. The $i$-th row in this symmetric matrix characterizes the connectivity-based degree of similarity of the $i$-th source with all the other sources within the $l$-th cluster. We chose to cluster rows of the connectivity-based correlation matrix rather than connectivity profiles themselves because this transforms the high dimensional space of connectivity profiles into a lower dimensional space of connectivity-based correlations. Furthermore two sources are clustered not only if their connectivity is similar but also if their connectivitybased degrees of similarity with all the other sources are close.

Thus, we performed a $k$-means algorithm on rows of

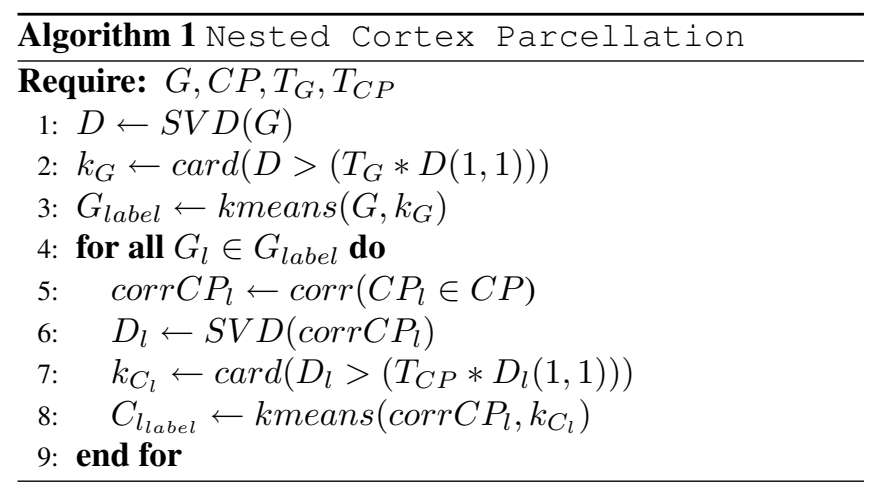

the $l$-th connectivity-based correlation matrix, Algorithm 1: line 8 . This produces a connectivity-based cortex parcellation nested in the lead field-based cortex parcellation which reveals $\sum_{l=1}^{L} k_{C_{l}}$ regions.

Like for any clustering method, the number of clusters has to be set. For this, we took a singular value decomposition (SVD) on data to cluster. We clustered data into the number of singular values above a threshold, $T_{G}$ for the lead field and $T_{C P}$ for the connectivity-based correlation matrix, of the principal singular value, given by the first entry of the diagonal matrix $D$ of the SVD, Algorithm 1: line 2 to 3 for the lead field, Algorithm 1: line 6 to 7 for the connectivity-based correlation matrix.

\section{RESULTS AND DISCUSSION}

We performed the nested parcellation methodology outlined on the flowchart on Fig. 1 on the ROI defined section 2.2.2. Taking a threshold value $T_{G}$ and $T_{C P}$ equal to $10 \%$, the two gyri touching the left central fissure were lead field-based parcelled into 68 clusters then into 586 clusters adding connectivity information, see Fig. 4.

Results show that lead field-based parcellation is close to what we could expect: sources with similar orientations and positioned close by are assembled into clusters. Lead field-based clusters generally form one connected component. Some of these clusters are made of two components, 


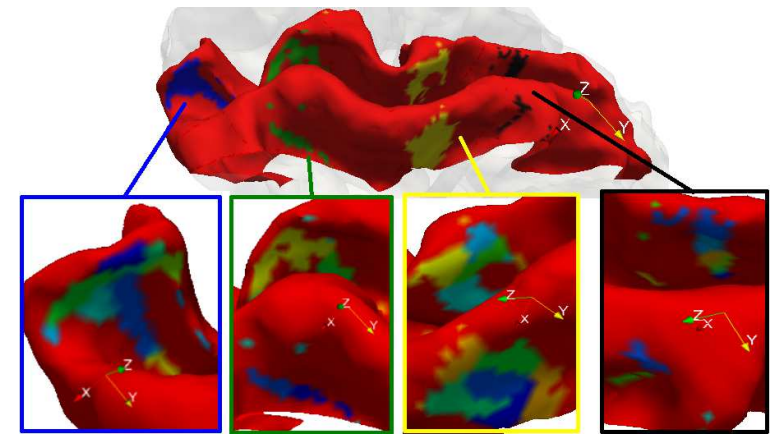

Fig. 4. 4 leadfield-based parcels out of 68 (blue, green, yellow and black). Zoom on the 4 clusters showing each one of their connectivity-based parcellations.

see Fig. 4: one on one gyrus, the second on the other gyrus. It does not contradict our expectations: sources of a cluster astride two gyri have the same orientation and are close in distance. The MEG inverse problem can not separate such sources. We can observe on Fig. 4 some tiny patches that can be ignored. Let us notice our nested cortex parcellation is automatic and do not have connexity constraints.

Nested parcellation illustrated on Fig. 4 shows subclusters that allow to refine the lead field-based clustering and generally delimitate the two gyri. However, some subclusters can be common to two gyri. This result can come from the fact that probabilist tractography combines shortrange connectivity (short-range fibers shaped with a $U$ form which connect one gyrus to its neighbour following the cortex, [8]) and long-range connectivity, these sub-clusters being linked by short-range connectivity. Thus the method may be a first step to analyze sources that are seeds of long-range connectivity or seeds of short-range connectivity.

\section{CONCLUSION}

Results generally show suitable clusters allowing a reduction of dimension for connectivity-based parcellation, and a new way to regularize the sources space for the MEG inverse problem.

At all events, this work gives us an interesting and useful tool to better understand the structure-function relationship. The study of the symmetry and the non-symmetry of the brain as performed in [10], could greatly benefit from our cortex parcellation to give important information about the correlations between structure and function. Finally, our nested cortex parcellation allow the use of structural benchmarks for the neural modelling. Our method is obviously useful for localization of sources in MEG but could be used for all others modalities measuring the functional activity in the brain.

\section{ACKNOWLEDGEMENTS}

The authors akcknowledge support from the ANR grant ViMAGINE and from the Regional Council of Provence Alpes Cote d'Azur.

\section{REFERENCES}

[1] P.E. Roland and K. Zilles, "Structural divisions and functional fields in the human cerebral cortex," Brain Res, vol. 26, pp. 87-105, 1998.

[2] D.S. Tuch and V.J. Wedeen T.G. Reese, M.R. Wiegell, "Diffusion mri of complex neural architecture," Neuron, vol. 40(5), pp. 885-895, 2003.

[3] T.E. Behrens, H. Johansen-Berg, M.W. Woolrich, S.M. Smith, C.A. Wheeler-Kingshott, and P.A. Boulby, "Non-invasive mapping of connections between human thalamus and cortex using diffusion imaging," Nature, vol. 6(7), pp. 750-757, 2003.

[4] A. Anwander, M. Tittegemeyer, and D. Von Cramon, "Connectivity-based parcellation of broca's area," Cerebral Cortex, pp. 816-825, 2007.

[5] P. Roca, D. Riviere, P. Guevara, C. Poupon, and J.F. Mangin, "Mapping human whole-brain structural networks with diffusion mri," MICCAI, vol. lncs 5761, 2009.

[6] L. Meng, J. Xiang, D Rose, R. Kotecha, J. Vannest, A. Byars, and T. Degrauw, "Time course and neural network for comparing written and spoken words: A meg and dti study," BIOMAG, vol. 28(11), pp. 338-341, 2010 .

[7] I.S. Mohamed, H. Otsuboa, M. Shroffb, E. Donnera, J. Drakec, and O. Carter, "Magnetoencephalography and diffusion tensor imaging in gelastic seizures secondary to a cingulate gyrus lesion," Clinical Neurology and Neurosurgery, vol. 109(2), pp. 182-187, 2007.

[8] A. Schuz and V. Braitenberg, "The human cortical white matter: Quantitative aspects of cortico-cortical longrange connectivity," Medical Image Analysis, 2002.

[9] S. Jbabdi H. Johansen-Berg T. Behrens, J.C Klein, Connectivity-Based parcellation of Gray Matter, 2010.

[10] M. Ellmore, M.S. Beauchamp, J.I. Breier, J.D. Slater, G.P. Kalamangalam, and T.J. ONeill, "Temporal lobe white matter asymmetry and language laterality in epilepsy patients," NeuroImage, vol. 49(3), 2010. 